\title{
Optimización en ubicación de estaciones base de baja potencia LTE
}

\author{
Yair Ali Cerecedo Torres, Miguel Sánchez Meraz, Antonio Valdovinos Bardají, \\ Ángela Hernandez Solana \\ Instituto Politécnico Nacional, Escuela Superior de Ingeniería Mecánica y Eléctrica, \\ Ciudad de México, México \\ Universidad de Zaragoza, Escuela de Ingeniería y Arquitectura , Zaragoza, \\ España
}

ycerecedot0900@alumno.ipn.mx, mmeraz@ipn.mx, toni@unizar.es, anhersol@unizar.es

Resumen. En este trabajo se desarrolla un Modelo de Programación Lineal Entera (MPLE) para encontrar el emplazamiento óptimo de Estaciones Base de Baja Potencia (EBBP) con el objetivo de solucionar problemas de congestión en una macro celda LTE. Una función de costos representa la función objetivo del modelo de optimización y está restringida a los parámetros técnicos de diseño más importantes en el proceso de planeación de redes: cobertura, demanda de usuario y capacidad. El MPLE es aplicado y evaluado en dos escenarios de análisis. Estos escenarios se configuran en un software de planificación de redes tanto para alimentar al modelo como para determinar si los requerimientos de los escenarios son alcanzados.

Palabras clave: Modelo de programación pineal entera, despliegue de estaciones base de baja potencia, planeación de redes.

\section{Optimization in Placement of Low Power Base Stations LTE}

\begin{abstract}
This paper develops an Integer Linear Programming Model (ILPM) to find the optimal emplacement of low power base stations in order to resolve traffic congestion problem in hotspots within a LTE macro cell. A cost function represents the objective function inside the ILPM and it is restricted to the most important technical parameters in the planning networks process of design: user demand, coverage and capacity. The ILPM is implemented and evaluated in two analysis scenarios. These scenarios are set up in planning network software to both feed the input of model and determine if the requirements of the scenario are met.
\end{abstract}

Keywords. Integer linear programming model, deployment of low power base stations, small cell, planning network. 


\section{Introducción}

Desde el punto de vista de capas, una manera de expandir y aumentar las capacidades de una red de acceso LTE mientras se mantiene como una red homogénea, es agregar más sectores o desplegando más Macro Estaciones Base (MEB) en el área de interés. Sin embargo, estas tareas sólo pueden llevarse a cabo en cierta medida debido a que el espectro es limitado y la búsqueda de nuevos emplazamientos es una tarea difícil sobre todo en entornos urbanos [1].

En las especificaciones de LTE avanzado (R10) se propone introducir Estaciones Base de Baja Potencia (EBBP) dentro del área de servicio de macro estaciones base para aumentar de manera más eficiente las capacidades de las redes de acceso LTE [3, 4]. Esto permite aumentar la capacidad de la red en zonas específicas donde se presentan grandes concentraciones de usuarios y/o cubrir áreas que no son cubiertas por las macro estaciones base debido a condiciones de propagación [2].

Un factor muy importante que determina un correcto despliegue es la elección de la ubicación de los emplazamientos donde se instalarán las estaciones base de baja potencia. En los últimos años se han realizado numerosos trabajos que se han apoyado con la investigación de operaciones para determinar el emplazamiento óptimo de EBBP. Dicha área ofrece la posibilidad de desarrollar modelos matemáticos de optimización que son útiles para determinar la ubicación óptima de estaciones base, minimizando o maximizando diferentes parámetros técnicos y económicos [3].

El trabajo se organiza de la siguiente manera: en el apartado 2 se describe el modelo de optimización desarrollado, en el apartado 3 se presentan los resultados de la aplicación del modelo de optimización en dos escenarios de análisis (EA) y, por último, el apartado 4 contiene las conclusiones generales de este trabajo [4, 5].

\section{Problema de optimización para la ubicación de estaciones base de baja potencia}

El desarrollo del modelo de optimización parte de un escenario de análisis general como se muestra en la Figura 1. Este escenario general consiste en una macro celda dividida en $l \in L$ sectores, que eventualmente presenta un número determinado de zonas de alta densidad de usuarios (ZAD) $h_{l, n} \in H$. En dichas zonas, se disponen de $p_{n}^{h \in H} \in P$ posibles emplazamientos (PE) para instalar EBBP y se disponen de $b \in B$ anchos de canal para ser asignados dentro de un espectro limitado. Estos canales se identifican con el conjunto $B=\left\{b_{0}, b_{1}, b_{2}, \ldots, b_{7}\right\}$ que corresponden a los anchos de canal disponibles en LTE [6]. El primer elemento de este conjunto $b_{0}$ está asociado a un canal de $0 \mathrm{MHz}$ y debe ser interpretado como sin canal asignado. Para realizar un despliegue ordenado de las EBBP, se proponen diferentes escenarios de despliegue (ED) identificados con el conjunto $F=\left\{f_{1}, f_{2}, f_{3}, \ldots, f_{n}\right\}$. Los ED $f \in F$ son evaluados en su desempeño a través de simulaciones en una herramienta computacional de 
planeación para determinar el área cubierta del área total $A_{T}$ de las ZAD $h \in H$ que se encuentren en el sector $l \in L$.El número de escenarios de despliegue $f \in F$ en cada $h \in H$ es determinado por $2^{p}-1$. Cada ED $f \in F$ tiene un costo de instalación $C_{f}$, de la misma manera, se tiene un costo asociado $C_{b}$ por la asignación del ancho de banda $b \in B$ tanto para las EBBP como para las sectorizaciones $l \in L$ de la macro celda.

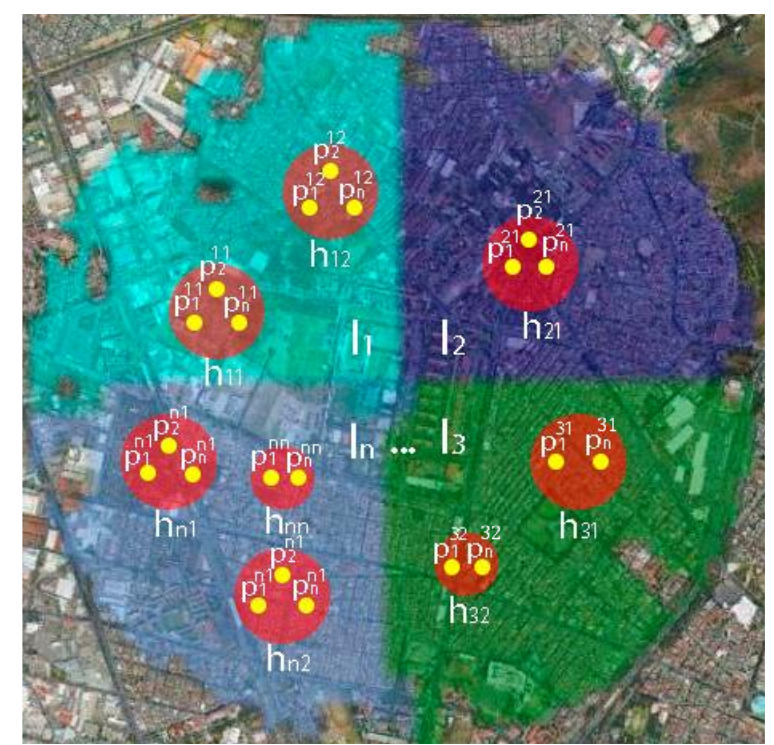

Fig. 1. Escenario general de análisis para el desarrollo del modelo de optimización lineal entero.

El problema de optimización además de determinar la posición de EBBP también consiste en realizar una gestión óptima de los recursos espectrales disponibles, es decir, asignar el menor ancho de banda posible tanto a los sectores $l \in L$ de la MEB como a cada una de las EBBP desplegadas, lo que conlleva a la minimización de los costos del despliegue.

\subsection{Variables de decisión}

Se introducen tres variables booleanas de decisión para el problema de optimización:

$$
\begin{aligned}
& x_{h, f}^{l}= \begin{cases}1 & \text { Se instala } f \in F \text { en } h \in H \text { ubicada en } l \in L \text { de la macro celda. } \\
0 & \text { Otro caso }\end{cases} \\
& y_{p, b}^{l, h}= \begin{cases}1 & \text { Se asigna } b \in B \text { a } \mathrm{p}_{n}^{h \in H} \in P \text { en } h \in H \text { ubicada en } l \in L \text { de la macro celda. } \\
0 & \text { Otro caso }\end{cases} \\
& z_{b}^{l}= \begin{cases}1 & \text { Se instala el canal } b \in B \text { al sector } l \in L \text { de la macro celda. } \\
0 & \text { Otro caso }\end{cases}
\end{aligned}
$$




\subsection{Función objetivo}

La función objetivo que se ha planteado para el problema de encontrar la ubicación óptima de las EBBP, es una función de costos que considera los siguientes aspectos: costo de instalación del despliegue de EBBP, costo de la asignación de canal a las EBBP, costo de la asignación de canal a los sectores de la MEB. La función objetivo se muestra en la Ecuación 4:

$$
\min \gamma=\sum_{l \in L} \sum_{h \in H} \sum_{f \in F} C_{f} * x_{h, f}^{l}+\sum_{l \in L} \sum_{h \in H} \sum_{p \in P} \sum_{b \in B} C_{b} * y_{p, b}^{l, h}+\sum_{l \in L} \sum_{b \in B} C_{b} * Z_{b}^{l} .
$$

\subsection{Restricciones}

Requerimiento de cobertura. Para asegurar que el $f \in F$ atienda al menos a un porcentaje determinado del área $(R C)$ de la ZAD $h \in H$ ubicada en el sector $l \in L$, se debe cumplir la Relación 5, Donde $S_{f, p}^{l, h}$ es la estimación de cobertura de cada ED:

$$
R_{1}: \sum_{f \in F} \sum_{p \in P} x_{h, f}^{l} * S_{f, p}^{l, h} \geq R C
$$

Asignación de canal a las EBBP desplegadas. La asignación del ancho de banda del canal está en función de la demanda de servicio que se tenga presente en la ZAD $h \in H$ ubicada en el sector $l \in L$ Esta demanda está asociada a la densidad de usuarios de la zona $U_{h}^{l}$ y su perfil de tráfico $T d_{h}^{l}$. La demanda que existe también depende del área $S_{f, p}^{l, h}$ que esté atendiendo la EBBP en el posible emplazamiento $p_{n}^{h \in H} \in P$ del ED $f \in F$ del área total de la ZAD.

Por otra parte, la capacidad que se alcanza en la ZAD $h \in H$ depende del número de bloques de recursos $W_{b}$ disponibles en el ancho de canal $b \in B$ asignado y del tipo de modulación del enlace $M s c_{h, f}^{l}$. Para asegurar que la demanda sea menor a la capacidad se debe cumplir la Relación 6:

$$
R_{2}: x_{h, f}^{l} * U_{h}^{l} * T d_{h}^{l} *\left(\frac{S_{f, p}^{l, h} * A_{h}^{l}}{100}\right) \leq \sum_{b \in B} \frac{120 * y_{p, b}^{l, h} * W_{b} * M s c_{h, f}^{l}}{10^{-3}}
$$

Asignación del canal a los sectores de la MEB. De la misma manera que el planteamiento de la asignación de canal a las EBBP, la asignación del canal a los sectores de la MEB depende de la demanda y de la capacidad de acuerdo, en general, a los usuarios atendidos dentro de la macro celda y al canal asignado a los sectores respectivamente. Sin embargo, se deben tomar en cuenta los usuarios que no sean atendidos por las EBBP. Para asegurar que la demanda sea menor a la capacidad en la MEB se debe cumplir la Relación 7: 


$$
\begin{aligned}
& R_{3}: U m_{l} * T d m_{l} *\left(\frac{A m_{l} * A_{T}}{100}-\sum_{h \in H} A_{h}^{l}\right)+U_{h}^{l} * T d_{h}^{l} * X_{h, f}^{l} *\left(A_{h}^{l}-\sum_{p_{h}^{h \in H} \in P} \frac{S_{f, p}^{l, h} * A_{h}^{l}}{100}\right) \ldots \\
& \ldots \leq \sum_{b \in B} \frac{120 * z_{b}^{l} * W_{b} * M s c}{10^{-3}} .
\end{aligned}
$$

El ancho de banda disponible es limitado. La suma de los anchos de canal asignados tanto a los sectores $l \in L$ de la macro celda como los asignados a las EBBP desplegadas, debe ser menor que el ancho de banda disponible por el operador como se muestra en la Relación 8.

$$
R_{4}: \sum_{b \in B} z_{b}^{l} * W_{b}+\sum_{b \in B} Y_{p, b}^{l, h} * W_{b} \leq W_{\text {disponible }}
$$

Existe un ED válido si existe un canal asignado. Para asegurar que se asigne un canal a cada EBBP instalada en el PE $p_{n}^{h \in H} \in P$ en las ZAD $h \in H$ ubicadas en el sector $l \in L$, se debe cumplir la Relación 9:

$$
R_{5}: x_{h, f}^{l} \leq \sum_{p_{h} \in H} \sum_{b \in B} y_{p, b}^{l, h} .
$$

Un ED en cada ZAD. Para asegurar que en la ZAD $h \in H$ sólo se asigne un ED $f \in F$ , se debe cumplir la Relación 10:

$$
R_{6}: \sum_{f \in F} x_{h, f}^{l} \leq 1
$$

Un canal asignado para cada EBBP desplegada. Se debe de asignar sólo un ancho de canal $b \in B$ para cada ED $f \in F$ en la ZAD $h \in H$ ubicada en el sector $l \in L$, para esto, se debe cumplir la Relación 11:

$$
R_{7}: \sum_{b \in B} Y_{p, b}^{l, h} \leq 1
$$

Un canal asignado para cada sector de la MEB. Para asegurar que sólo se asigne un canal $b \in B$ en cada sector $l \in L$ de la MEB, se debe cumplir la Relación 12:

$$
R_{8}: \sum_{b \in B} z_{b}^{l} \leq 1
$$

\section{Resultados de la aplicación del modelo de optimización}

\subsection{Escenario de análisis A (EA A)}

\section{Planteamiento}

El modelo es aplicado a una MEB que está operando con una antena omnidireccional transmitiendo a una potencia de $49.5 \mathrm{dBm}$. Esta MEB se encuentra instalada a una 
altura de 25 metros. Con base en la recopilación de información técnica del equipo el problema se traslada a la herramienta computacional de planeación de redes Mentum Planet [7] para determinar el área de análisis de la macro celda mediante un análisis de RSRP como se muestra en la Figura 2. Cabe mencionar que la frecuencia de operación para los dos escenarios de análisis se encuentra en la banda APT $700 \mathrm{MHZ}$. Se propone un esquema de modulación promedio de 3 bits*símbolo con base a una densidad de usuarios uniforme $U m_{l}$ de 50 usuarios por $\mathrm{Km}^{2}$ [4].

El área de análisis resultante es una macro celda de 800 metros de radio que eventualmente presenta tres ZAD $h_{1,1}, h_{1,2}, \mathrm{y} h_{1,3}$. Se disponen de dos PE $p_{1}^{h}$ y $p_{2}^{h}$ para satisfacer la demanda en cada una de estas zonas como se muestra en la Figura 3. Se requiere un $95 \%$ de cobertura en las ZAD.

De acuerdo a los dos posibles emplazamientos disponibles en cada ZAD existe un total de tres ED de EBBP, $F=\left\{f_{1}, f_{2}, f_{3}\right\}$. Se propone una eficiencia de modulación promedio con base en el número de EBBP consideradas para desplegar [4]. La densidad de usuarios $U_{l}^{h}$ es uniforme y se establece para este escenario de 500 usuarios por $\mathrm{Km}^{2}$, para las tres ZAD definidas. Además, se define un requerimiento de cobertura en estas zonas de $\mathrm{RC} \geq 95 \%$. El ancho de banda disponible es de $90 \mathrm{MHz}$.

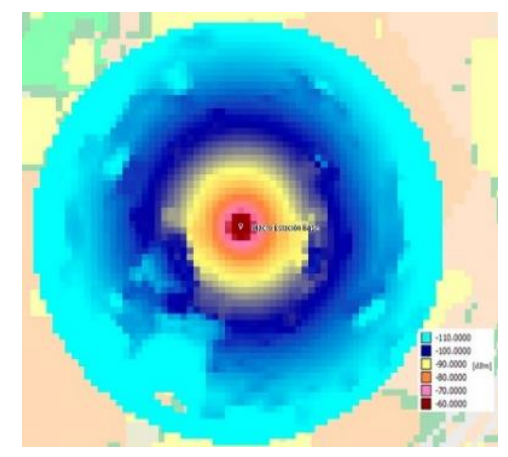

Fig. 2. Definición del área de análisis de la macro celda en el escenario de análisis A.

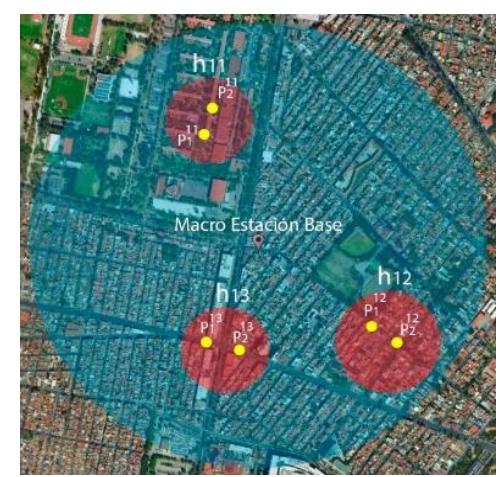

Fig. 3. Zonas de alta demanda (zonas rojas) y posibles emplazamientos (puntos amarrillos). 
En este escenario los costos por instalar una EBBP en los PE $p_{1}^{h}$ y $p_{2}^{h}$ son de 2,000 y 4,000 unidades genéricas de valor respectivamente, para las tres ZAD. Por otra parte, el costo por el ancho de banda de cada canal es $C_{b}=\{0,1,2,3,4,5,6\} * 1000$.

\section{Resultados}

El valor de la función objetivo tiene un valor de $\gamma=31,000$ [UV]. Cabe mencionar que el modelo de optimización se resuelve en la herramienta computacional CPLEX ILOG [8]. El despliegue optimizado resultante del modelo de optimización se muestra en la Figura 4. Como se puede observar se habilitaron los emplazamientos $p_{1}^{11}$, $\left(p_{1}^{12}\right.$ y $\left.\mathrm{p}_{2}^{12}\right)$ y $\left(p_{1}^{13}\right.$ y $\left.\mathrm{p}_{2}^{13}\right)$ en las zonas $h_{1,1}, h_{1,2}$ y $h_{1,3}$ respectivamente. Además, los canales que se habilitaron son $10 \mathrm{MHz}$ para $p_{1}^{11}, 5$ y $3 \mathrm{MHz}$ para $p_{1}^{12}$ y $\mathrm{p}_{2}^{12}$ respectivamente y un canal de $3 \mathrm{MHz}$ para cada emplazamiento $p_{1}^{13}$ y $\mathrm{p}_{2}^{13}$.

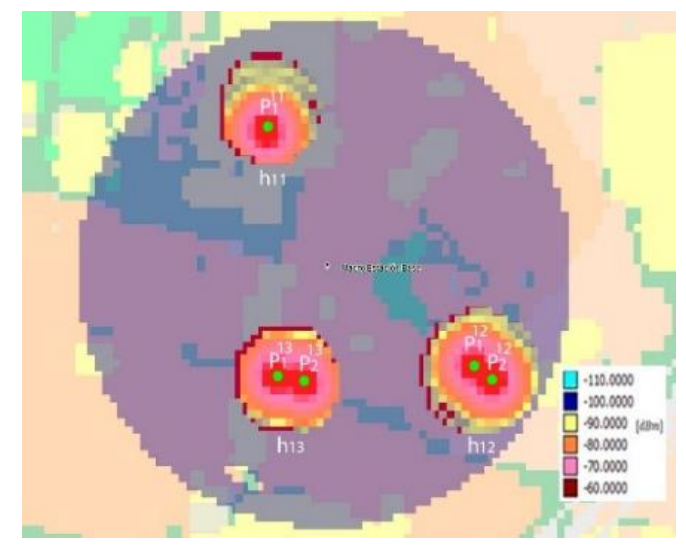

Fig. 4. Ubicación óptima de las EBBP (Despliegue optimizado).

En la Tabla 1 se demuestra que con este despliegue de EBBP se cumple que la demanda es menor a la capacidad en las ZAD.

Tabla 1. Análisis de capacidad en las zonas de alta demanda.

\begin{tabular}{ccccc}
\hline Zona & $\begin{array}{c}\text { Demanda } \\
\text { total }[\mathrm{Kbps}]\end{array}$ & $\begin{array}{c}\text { Canal } \\
\text { asignado. } \\
{[\mathrm{MHz}]}\end{array}$ & $\begin{array}{c}\text { Capacidad alcanzada con } \\
\text { el canal asignado }[\mathrm{Kbps}]\end{array}$ & $\begin{array}{c}\text { Capacidad total en } \\
\text { la zona. }\end{array}$ \\
\hline$h_{11}$ & $11,053.09$ & 10 & 12,000 & 12,000 \\
$h_{12}$ & $14,865.53$ & 5 & 12,000 & 19,200 \\
$h_{13}$ & $11,128.09$ & 3 & 7,200 & 14,400 \\
\hline
\end{tabular}

Por otra parte, el análisis a nivel macro celular consiste en determinar la demanda y la capacidad en los sectores de la macro celda antes y después de desplegar EBBP. El análisis se observa en Tabla 2. 
Yair Ali Cerecedo Torres, Miguel Sánchez Meraz, Antonio Valdovinos Bardají, et al.

Tabla 2. Análisis de capacidad en la macro celda.

\begin{tabular}{cccccc}
\hline Sector & $\begin{array}{c}\text { Demanda } \\
\text { sin EBBP } \\
{[\mathrm{Kbps}]}\end{array}$ & $\begin{array}{c}\text { Demanda } \\
\text { con EBBP } \\
\text { desplegadas } \\
{[\mathrm{Kbps}]}\end{array}$ & $\begin{array}{c}\text { Demanda } \\
\text { liberada } \\
{[\mathrm{Kbps}]}\end{array}$ & $\begin{array}{c}\text { Canal } \\
\text { asignado por } \\
\text { el modelo } \\
{[\mathrm{MHz}]}\end{array}$ & $\begin{array}{c}\text { Capacidad } \\
\text { alcanzada con } \\
\text { el canal } \\
\text { asignado } \\
{[\mathrm{Kbps}]}\end{array}$ \\
\hline$l_{1}$ & $60,616.67$ & $23,570.96$ & $\begin{array}{c}37,045.74 \\
61.11 \%\end{array}$ & 15 & 27,000 \\
\hline
\end{tabular}

La diferencia entre la demanda antes y después del despliegue de EBBP es del $61.11 \%$ y equivale a liberar a la macro estación base (no sectorizada) de $37 \mathrm{Mbps}$. Por otra parte, con el canal asignado se alcanza una tasa de transferencia de datos de 27,000 Kbps. Por lo tanto, se satisface la demanda requerida.

\subsection{Escenario de análisis B (EA B)}

\section{Planteamiento}

Se propone una MEB que está operando con con tres sectores de $120^{\circ}$, transmitiendo a una potencia (PIRE) de $60.50 \mathrm{dBm}$ y se encuentran a una altura de 25 metros. El resultado del análisis de RSRP realizado en la herramienta de planeación define una celda de $900 \mathrm{~m}$ de radio. Se propone un esquema de modulación promedio de 3 bits*símbolo con base a una densidad de usuarios uniforme $U m_{l}$ de 100 usuarios por $K m^{2}$ en cada sectorización de la macro celda. Se identifica una ZAD $h_{1,1}, h_{2,1}$ y $h_{3,1}$ para $l_{1}, l_{2}$ y $l_{3}$ respectivamente, como se muestra en la Figura 5. Cabe mencionar que en cada zona se presenta un esquema de modulación promedio de 3 bits*símbolo y una densidad de usuarios uniforme de 500 usuarios por $\mathrm{Km}^{2}$. Adicionalmente se requiere un $80 \%$ de cobertura en estas zonas. El ancho de banda disponible es de $90 \mathrm{MHz}$.

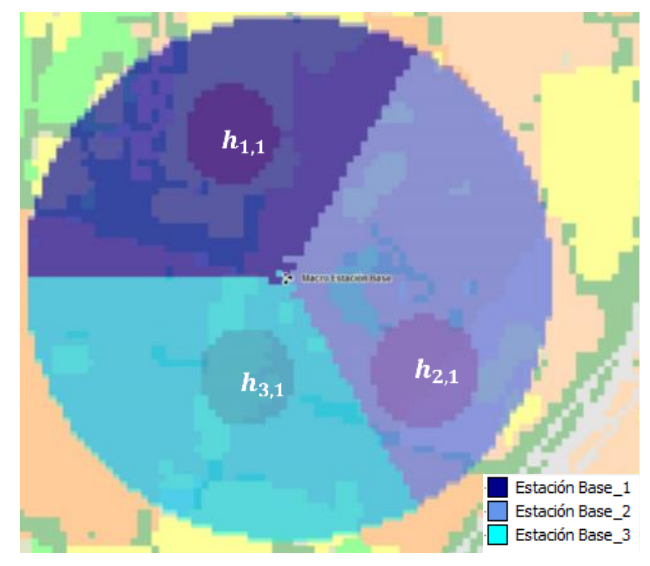

Fig. 5. Ubicación de zonas de alta demanda en los sectores de la macro celda. 


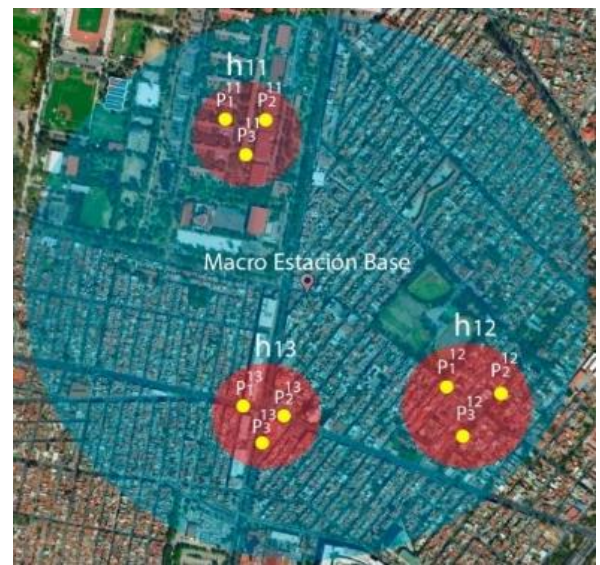

Fig. 6. Zonas de alta demanda (zonas rojas) y posibles emplazamientos (puntos amarrillos).

Para satisfacer la demanda en las ZAD se dispone, tres PE $p_{1}^{h}, p_{2}^{h}$ y $p_{3}^{h} \forall h \in H$ para desplegar EBBP como se muestra en la Figura 6. De acuerdo a los tres PE disponibles en cada ZAD existe un total de siete escenarios de despliegue de EBBP, $F=\left\{f_{1}, f_{2}, f_{3}, \ldots, f_{7}\right\}$. Se propone una eficiencia de modulación promedio con base en el número de EBBP consideradas para desplegar [4]. En este escenario los costos por instalar una EBBP en todos los PE es de $\{1,000$ unidades genéricas de valor. El costo por el ancho de banda de cada canal es el mismo del escenario anterior.

\section{Resultados}

El valor de la función objetivo tiene un valor de $\gamma=31,000$ [UV]. El despliegue optimizado resultante del modelo de optimización se muestra en la Figura 7.

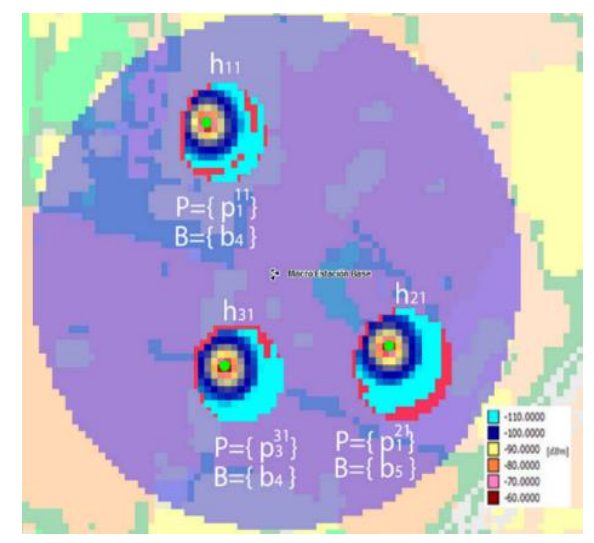

Fig. 7. Ubicación óptima de las EBBP (Despliegue optimizado).

Como se puede observar se habilitaron los emplazamientos $p_{1}^{11}, p_{1}^{21}$ y $p_{3}^{31}$ para $h_{1,1}$, $h_{2,1}$ y $h_{3,1}$ respectivamente. Además, los canales que se habilitaron para dichos 
emplazamientos son $10 \mathrm{MHz}, 15 \mathrm{MHz}$ y $10 \mathrm{MHz}$. En la Tabla 3 se muestra que con este despliegue de EBBP se cumple que la demanda es menor a la capacidad en las ZAD.

Table 3. Análisis de capacidad en las zonas de alta demanda.

\begin{tabular}{cccc}
\hline Zona & $\begin{array}{c}\text { Demanda total } \\
{[\mathrm{Kbps}]}\end{array}$ & $\begin{array}{c}\text { Canal } \\
\text { asignado } \\
{[\mathrm{MHz}]}\end{array}$ & $\begin{array}{c}\text { Capacidad alcanzada con el canal } \\
\text { asignado }[\mathrm{Kbps}]\end{array}$ \\
\hline$h_{1,1}$ & $10,159.09$ & 10 & 12,000 \\
$h_{2,1}$ & $12,103.63$ & 15 & 18,000 \\
$h_{3,1}$ & $9,990.91$ & 10 & 12,00 \\
\hline
\end{tabular}

Por otra parte, el análisis a nivel macro celular consiste en determinar la demanda y la capacidad en los sectores de la macro celda antes y después de desplegar EBBP. El análisis se observa en Tabla 4.

Table 4. Análisis de capacidad en la macro celda.

\begin{tabular}{cccc}
\hline Sector & $l_{1}$ & $l_{2}$ & $l_{3}$ \\
\hline Demanda sin EBBP desplegadas & $31,852.57$ & $35,568.62$ & $32,418.13$ \\
{$[\mathrm{Kbps}]$} & $21,693.48$ & 23,465 & $22,490.22$ \\
Demanda con EBBP desplegadas & $10,159.08$ & $12,103.63$ & $9,990.91$ \\
{$[\mathrm{Kbps}]$} & $31.89 \%$ & $34.03 \%$ & $30.76 \%$ \\
Demanda liberada $[\mathrm{Kbps}]$ & 15 & 15 & 15 \\
Canal asignado por el modelo & 27,000 & 27,000 & 27,000 \\
\hline $\mathrm{MHz}]$ & & & \\
\hline Capacidad alcanzada $[\mathrm{Kbps}]$ &
\end{tabular}

La diferencia entre la demanda antes y después del despliegue de EBBP es la demanda que es atendida por las zonas de alta demanda en cada sector y equivale, desde este punto de vista, a liberar a los sectores $l_{1}, l_{2}$ y $l_{3}$ del $31.89 \%, 34.03 \%$ y $30.76 \%$ de su carga respectivamente. Por otra parte, con el canal asignado a cada uno de los sectores, se alcanza una tasa de transferencia de datos de 27,000 Kbps. Por lo tanto, en todos los sectores de la macro celda se satisface la demanda requerida.

\section{Conclusiones}

En este trabajo se desarrolla un modelo de optimización lineal entera para mejorar el desempeño en el servicio de una red de comunicaciones móviles LTE a través del despliegue de EBBP. La función objetivo toma un valor de 31,000 [UV] para los dos EA. Cabe mencionar que se habilitaron más EBBP en el EA A con respecto al EA B pero el espectro utilizado es de $39 \mathrm{MHz}$ y $85 \mathrm{MHz}$ respectivamente. Utilizar menor 
espectro representa un menor costo económico. El espectro utilizado es menor debido a que el RC es mayor en el EA A con respecto al EA B y la densidad de usuarios en las ZAD es menor en el EA A con respecto al EA B. La solución del modelo de optimización es encontrar la configuración que satisfaga estos requerimientos con el mínimo de recursos económicos. Otro escenario de análisis es una macro celda sectorizada donde se presentan más de una ZAD en cada sector. Esté planteamiento es resuelto replicando el escenario de análisis A para las sectorizaciones correspondientes. El objetivo es demostrar que la contribución desarrollada en este trabajo puede ser aplicado a macro celdas con cualquier tipo de configuración. En futuros trabajos se considerará el manejo de interferencias aplicando como primera etapa el esquema ICIC (Inter-Cell Coordination Interference) y como segunda etapa el esquema eICIC (Enhanced Inter-cell Coordination Interference).

Agradecimientos. Los autores agradecen al Instituto Politécnico Nacional y a la Universidad de Zaragoza por el apoyo brindado para la realización de este trabajo.

\section{Referencias}

1. M. Ergen: Mobile Broadband, Including WiMAX and LTE. Springer (2009)

2. H. Holma, A. Toskala, J. Reunanen: LTE Small Cell Optimization, 3GPP Evolution to Release 13. Wiley (2016)

3. Y. Park, J. Heo, H. Kim, H. Wang, S. Choi, T. Yu, D. Hong: Effective Small Cell Deployment with Interference and Traffic Consideration. IEEE Commun. Mag. (2014)

4. Y. A. Cerecedo: Optimización en ubicación de estaciones de baja potencia lte. Tesis, Instituto Politécnico Nacional (2016)

5. Y. A. Cerecedo, M. Sánchez, G. Santillán: Optimización de cobertura y capacidad en el diseño de redes WiMAX. Tesis, Instituto Politécnico Nacional (2013)

6. A. Ghosh, J. Zhang, J. G. Andrews, R. Muhamed: Fundamentals of LTE. Prentice Hall. (2011)

7. M. Planet, C. Planning, T. Academy: Coverage Planning using Mentum Planet Creating a Project.

8. Ibm, IBM ILOG CPLEX Optimization Studio, User’s Manual (2014) 